\title{
Conducting Clinical Trials in the Time of a Pandemic
}

\author{
Joal D. Beane, MD, Priya H. Dedhia, MD, PhD, Aslam Ejaz, MD, MPH, Carlo M. Contreras, MD, \\ Jordan M. Cloyd, MD, Allan Tsung, MD, and Timothy M. Pawlik, MD, MPH, PhD四
}

Keywords: academic mission, clinical trials, COVID-19, guidelines, pandemic, research

(Ann Surg 2020;272:e219-e221)

Trhe he COVID-19 pandemic has created formidable challenges to the conduct of clinical trials and research. Overcoming these challenges has required creativity and resourcefulness to mount broadsweeping changes. Responses to mitigate the effects of the pandemic have included: (1) the development of strategies to support research programs during unforeseen economic loss, (2) establishment of institutional guidelines for clinical trials, (3) measures to ensure a healthy clinical research team, (4) use of innovative technologies to maintain access to clinical trials, (5) amendment of protocols to avoid costly trial closures, and (6) the strategic reopening of suspended clinical trials. Herein we discuss how each of these strategies has been used to sustain clinical trials and surgical research in the face of extraordinary adversity.

\section{IMPACT OF FINANCIAL HARDSHIPS ON CLINICAL TRIALS}

The COVID-19 pandemic has caused significant financial ramifications to all stakeholders in clinical trials - pharmaceutical and device companies, patients, and healthcare systems. As such, institutional leadership may need to reprioritize clinical research efforts and shift resources away from high-risk pilot studies and phase I trials to support more advanced phase III studies. In addition, increasing efforts in grant submissions, other public and private funding, and charitable donations are needed to boost funds to support clinical research. Although the economic impact will be felt for years and is well beyond the control of the academician and clinical trialist, using the principles outlined below to reduce costly errors and to preserve ongoing trial efforts may be our best chance to mitigate the effects of the COVID-19 pandemic.

\section{MANAGEMENT OF CLINICAL TRIALS DURING A PANDEMIC: ESTABLISHING A PLAYBOOK}

Institutional guidelines should be established relative to initiating new clinical trials, managing accrual efforts to current studies, and discerning between essential and nonessential studies. Patient safety must remain top priority and a reappraisal of ongoing clinical trials is vital to ensure appropriate balance of risks and benefits to the

\footnotetext{
From the Division of Surgical Oncology, Department of Surgery, The Ohio State University Wexner Medical Center, Columbus, Ohio.

\Tim.Pawlik@osumc.edu.

Contributions: (I) Conception and design: Beane, Dedhia, Contreras, Ejaz, Pawlik; (II) Administrative support: TM Pawlik; (III) Collection and assembly of data: Beane, Dedhia, Contreras, Ejaz, Carson, Pawlik; (IV) Data analysis and interpretation Beane, Dedhia, Contreras, Ejaz, Pawlik; (V) Manuscript writing: All authors; (VI) Final approval of manuscript: All authors.

The authors report no conflicts of interest.

Copyright (C) 2020 Wolters Kluwer Health, Inc. All rights reserved.

ISSN: 0003-4932/20/27203-e219
}

DOI: $10.1097 /$ SLA.0000000000004114 patient and the health system. Navigating these types of decisions can be difficult and errors in judgement can be costly. Decisions should include all stakeholders and be informed by guidelines published by the Food and Drug Administration. ${ }^{1}$ In addition to these guidelines and frequent updates from the institutional review board (IRB), our institution has established a monitoring team that is readily available to review evolving research decisions. Specifically, an IRB "hotline" has been established to ensure accessibility to this team. Experienced researchers and clinical trialists are available to help navigate unexpected challenges and ensuring patient safety and trial integrity.

\section{PRESERVING THE HEALTH AND SAFETY OF THE CLINICAL RESEARCH TEAM}

Maintaining the wellbeing of the clinical research workforce is paramount to preserving clinical trials during a pandemic. However, the availability of staff can decrease by as much as $40 \%-50 \%$ due to illness, death, absenteeism related to fear and anxiety, and competing needs such as caring for children or ill family members. ${ }^{2}$ One of the most important strategies to help preserve the workforce is to ensure adherence to national and international guidelines regarding the use of PPE. ${ }^{3}$ In addition to physical safety, measures to ensure mental fitness are just as important to ensuring the research team's health and wellbeing. Although many centers have already experienced their surge of COVID-19 infections and the clinical burden is waning, the psychological impact of the pandemic may linger. ${ }^{2,4}$ In addition, as clinical volume and research begin to ramp back up, a strained workforce may result in extended hours for clinical staff. As such, it is imperative that employees are provided with resources to safeguard mental health (Fig. 1). ${ }^{4}$ These strategies may be helpful for nonclinical research staff, and individuals who may be anxious about the timing of laboratory reopenings and/or the ability to obtain research funding or grant extensions.

\section{MAINTAINING ACCESS TO CLINICAL TRIALS DURING A PANDEMIC}

At our institution, telemedicine is being used to interface with patients to discuss diagnoses, review imaging, and provide multidisciplinary consultation. In addition, telemedicine is being employed to contact trial participants, enroll and screen patients, and assess the status of patients already accrued in clinical trials. In March, over the course of 1 week, the volume of telemedicine visits jumped from less than 100 encounters per day to well over 2200 per day. In addition to maintaining access to clinical trials, the improved communication afforded by telemedicine has aided in reassuring participants that their involvement remains important during this time, which has helped to prevent costly dropouts or nonadherence. ${ }^{5}$ Recently, telemedicine has also been approved as a means to have discussions regarding informed consent as long as a document that can be mailed to and signed by the patients is completed. These benefits have resulted in an exponential increase in telemedicine utilization that have been invaluable to preserving access to clinical trials and research and to capturing important data points with regards to efficacy and adverse events that otherwise would have been lost. 


\section{COPE with COVID}

\section{C ontrol the things that you can, not the things you can't \\ O pen up and share your feelings \\ $\boldsymbol{P}$ ractice daily stress reduction tactics, including physical activity \\ $\boldsymbol{E}$ ngage in mindfulness; be here now; worry will not help!}

C ount your blessings daily

O verturn negative thoughts to positive

$\boldsymbol{V}$ olunteer to help others

I dentify helpful supports and resources

D o your part to prevent spread of the virus

- Bern Melnyk

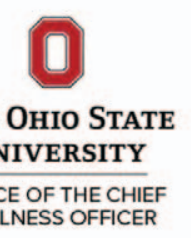

FIGURE 1. Sample of a business card-type resource provided to staff to assist with the psychological impact of COVID-19.
Although technology and telemedicine platforms may improve access to care and clinical trials, the availability of specific devices or implants may threaten continuity of certain trials. For essential clinical trials, investigators should work closely with the trial sponsor and device manufacturer. In addition, for multicenter studies excess inventory at 1 center can be shared with other participating centers to ensure that equitable trial accessibility is maintained.

\section{AMENDING CLINICAL TRIALS TO WEATHER THE STORM}

Efforts to modify protocols to comply with the emergency public health response and the guidelines established by the Food and Drug Administration and IRB are encouraged. Investigators need to prioritize collection of data, focusing on the primary endpoint and important secondary endpoints to remain in compliance. Clinical protocols should be reexamined thoroughly and amendments should be made to reduce superfluous clinical visits. Although many protocol changes typically required an amendment and lengthy review process, to avoid costly delays, reviews of amendments at our institution are being waived or expedited if the adjustment pertains to patient safety in the setting of COVID-19. For other modifications, a more formalized process is generally necessary, but should be expedited when possible.

Amendments should be used primarily to protect patients and research staff, but can also be used strategically to adapt protocols and avoid trial suspension. In this way, investigators may be able to maintain accrual of new patients or continue to collect important data from currently enrolled patients. For example, an amendment to monitor remotely an implanted device may facilitate continued treatment and collection of important data when nonessential interactions must be suspended. Alternatively, for patients receiving infusion therapy, an amendment can be written that allows the medication to be administered by a home health nurse to avoid crowded outpatient infusion centers. Protocols should be updated with ongoing data collection. For example, collecting patientreported endpoints for device or implant studies may include mailed or telephone surveys to replace scheduled in-person visits. ${ }^{5}$ In summary, adapting protocols to deliver an intervention or collect valuable outcomes can help to avoid costly suspensions, and updating trial procedures to ensure patient safety is important to mitigate the challenges posed by the COVID-19 pandemic.

\section{MANAGING PROTOCOL DEVIATIONS}

Given the current environment, IRBs should be prepared for a significant increase in protocol deviations. At our institution we are actively increasing the capacity of the IRB to review and address deviations expeditiously because a timely review is important to preserving trial integrity. To this end, deviations to clinical protocols should be tracked carefully, and documented, reported, and dealt with according to institutional guidelines. Guidelines distributed by the Department of Health and Human Resources help to define protocol deviations. For any type of deviation, thorough documentation in the medical record is vital to maintaining trial integrity. It is important to note that major protocol violations may not result in a corrective action plan or even a protocol amendment if related to challenges posed by the COVID-19 pandemic.

\section{WHEN TO SUSPEND A CLINICAL TRIAL}

A thorough reappraisal of all trials should be conducted regularly to reassess the development of new risks to participants, the availability of clinical staff and of the investigational drug or device, and disruptions related to quarantines, site closures, or travel limitations (Table 1). ${ }^{1}$ A reduction in clinical research staff may force clinical trials and other studies to be suspended. In addition, multiple, major deviations in study protocol or errors in data collection related to the pandemic may lead to a failed clinical trial, or worse, erroneous conclusions. As such, one may need to consider suspending a clinical trial until conditions improve. ${ }^{6}$ Thoughtful, timely appraisals and a

TABLE 1. Important Considerations in Deciding When to Suspend a Clinical Trial During the COVID-19 Pandemic ${ }^{1}$

Important considerations in deciding when to suspend a clinical trial during the COVID-19 pandemic

Safety risks to participants posed by the trial

Availability of clinical investigator, sub investigators, or support staff

Adequacy of current communications with the Institutional Review Board

(IRB) or Data Monitoring Committee (DMC)

Availability of equipment and materials for patient care

Availability of investigational drug or device

Presence of quarantines

Assessing for site closures

Travel restrictions

Federal and State public health measures 
multidisciplinary approach to these decisions can help avoid costly failures and ensure the safety of patients and providers, and the integrity of the trial, remain intact.

\section{A ROADMAP TO REOPENING CLINICAL TRIALS}

Now that many centers are beyond the anticipated surge, guidelines should be established for reopening clinical trials that were suspended during the peak response to COVID-19. Disease site leaders should work with treating physicians, research teams, clinical trial offices, and IRB leadership to create a succinct list of feasible studies that could be reactivated. The should be limited to essential trials that provide needed treatment for patients with limited alternatives. Trials of lower complexity and research-only procedures should be prioritized. Careful consideration should be given to whether more complex studies that involve extensive visits to the medical center or those trials that pose a challenge to social distancing should be included. In addition, only studies in which the protocol calendar can be followed without excessive deviation should be considered. When prioritizing trials, investigator-initiated studies may be more desirable because these studies can be amended more readily to permit changes in procedures and thus avoid protocol deviations. The ramp-up period must provide ample time for coordinators and the research team to begin returning to campus as needed and for acquiring the necessary approval from sponsors to begin enrolling again. To ensure safety and avoid costly errors, the reopening of clinical trials should occur in phases, based on priority, and within the guidelines established for social distancing.

\section{CONCLUSIONS}

In summary, the COVID-19 pandemic has created many unforeseen challenges that threaten important and costly clinical trials and research efforts. Although the impact of this pandemic is broad, the research team must remain dedicated to creating innovative approaches to advance clinical research for our patients.

\section{REFERENCES}

1. United States Food and Drug Administration. FDA Guidance on Conduct of Clinical Trials of Medical Products during COVID-19 Pandemic. 2020. Available at: fda.gov/media/136238/download. Accessed March 18, 2020.

2. Madhav N, Oppenheim B, Gallivan M, et al. Pandemics: risks, impacts, and mitigation. In: rd, Jamison DT, Gelband H, et al, eds. Disease Control Priorities: Improving Health and Reducing Poverty. Washington (DC); 2017.

3. Rajakaruna SJ, Liu WB, Ding YB, et al. Strategy and technology to prevent hospital-acquired infections: lessons from SARS, Ebola, and MERS in Asia and West Africa. Mil Med Res. 2017;4:32.

4. Cullen W, Gulati G, Kelly BD. Mental health in the Covid-19 pandemic. QJM. 2020;113:311-312.

5. McDermott MM, Newman AB. Preserving clinical trial integrity during the coronavirus pandemic. JAMA. 2020.

6. Moore TJ, Zhang H, Anderson G, et al. Estimated costs of pivotal trials for novel therapeutic agents approved by the US Food and Drug Administration, 2015-2016. JAMA Intern Med. 2018;178:1451-1457. 